\title{
Reliability and Convergence of Approach/Avoidance Bias Assessment Tasks in the Food Consumption Domain
}

\author{
[Stage 1 Registered Report] \\ Julian Basanovic, Laura Dondzilo, Daniel Rudaizky, Bram Van Bockstaele \\ Centre for the Advancement of Research on Emotion, \\ School of Psychological Science, \\ The University of Western Australia
}

\section{Authors note:}

All authors contributed equally to the work described in this manuscript and its publication.

Corresponding author:

Julian Basanovic; julian.basanovic@uwa.edu.au

Word count:

4051 words

Acknowledgements:

The research reported in this manuscript was supported by an Australian Research Council Grants FL170100167 and DP170104533

\section{Publication:}

This manuscript has passed peer review and has been issued an in principle acceptance (IPA) by the Quarterly Journal of Experimental Psychology, indicating that the article will be published pending successful completion of the study according to the exact methods and analytic procedures outlined, as well as a defensible and evidence-bound interpretation of the results. 


\begin{abstract}
Theories of motivation posit that people will more readily approach positive or appetitive stimuli while they are more likely to avoid negative or aversive stimuli. In recent years, there has been a growing interest in the relationship between biases in approach and avoidance behaviours for food cues and food craving and consumption behaviour. Two paradigms commonly employed by research to investigate this relationship are the Approach Avoidance Task (AAT) and the Stimulus Response Compatibility Task (SRCT). However, it is yet to be determined whether the measures yielded by these tasks reflect the same processes operating in the food craving and consumption domain. The purpose of the present study will be to address whether the AAT and SRCT paradigms provide internally reliable and convergent measures in their assessment of approach/avoidance bias to healthy and unhealthy food stimuli, and whether measures of approach/avoidance biases to healthy and unhealthy food yielded by the AAT and SRCT paradigms demonstrate comparable associations with individual differences in food craving and eating behaviour. The study will require participants to complete an SRCT, and two task variants of the AAT, and an estimate of participants' approach bias towards unhealthy food relative to healthy food will be computed from each. Analyses will determine the internal reliability of each of the approach bias scores, the degree to which the approach bias scores show convergent validity, and the degree to which the approach bias scores from each task are concurrently associated with individual differences in food craving and eating behaviour.
\end{abstract}




\section{Reliability and Convergence of Approach/Avoidance Bias Assessment Tasks in the Food Consumption Domain}

Theories of motivation posit that people will more readily approach positive or appetitive stimuli while they are more likely to avoid negative or aversive stimuli. For instance, in Lang's (Lang, 1995) theorizing, affect is driven by two primary motivational systems: An appetitive system, characterized by behavioural approach of positive or desired stimuli, and an aversive system, characterized by behavioural avoidance of negative or undesired stimuli. In a similar vein, the hierarchical model of approach-avoidance motivation (Elliot, 2006) posits that people automatically evaluate the valence of stimuli, and these evaluations in turn activate a tendency to approach positive or beneficial stimuli and a tendency to avoid negative or harmful stimuli. The relative tendency to more quickly execute movements that result in the approach of stimuli, as compared to the avoidance of stimuli, is typically referred to as an 'approach bias'. The inverse pattern is typically referred to as an 'avoidance bias'. To evaluate the degree to which an individual exhibits an approach bias, or avoidance bias, for a particular category of stimuli, the bias is computed across distinct categories of stimuli and the relative magnitude of the bias for each category of stimulus is assessed.

Early research into approach and avoidance biases observed that individuals were faster to execute movements that resulted in the approach of pleasant stimuli as compared to movements that resulted in the approach of unpleasant stimuli. In contrast, participants were also observed to be faster to execute movements that resulted in the avoidance of negative stimuli as compared to positive stimuli (Solarz, 1960). Likewise, Chen and Bargh (1999), demonstrated that individuals were faster at pulling a lever towards themselves when presented with positive words as compared to negative words on a computer screen, 
and were faster at pushing a lever when presented with negative words as compared to positive words.

In recent years there has been a growing interest in the relationship between approach biases for food stimuli and food craving and consumption behaviour. However, recent reviews of the field show that this relationship is somewhat inconsistent (Fricke \& Vogel, 2020; Kakoschke et al., 2019; Loijen et al., 2020). For instance, while Brockmeyer et al. (Brockmeyer et al., 2015) revealed a positive association between approach bias towards food stimuli and food craving, other studies could not reliably replicate this effect (Lender et al., 2018). As a further indication of the conflicting findings, while some studies revealed that overweight and obese individuals, but not healthy-weight individuals, were faster to approach than to avoid food stimuli (Kakoschke et al., 2017b; Mehl et al., 2018), other studies could not replicate this effect and some studies even found avoidance of food in obese individuals (Paslakis et al., 2017; Schmidt et al., 2018). Given that food-related approach/avoidance biases are proposed to be causally involved in the development and maintenance of eating and weight disorders (for reviews, see Kakoschke et al., 2019, Kemps, \& Tiggemann, 2017a; Loijen et al., 2020) it is important that the inconsistency in findings linking such biases to food consumption is resolved.

One explanation for these inconsistent findings lies with tasks that have been used to measure approach/avoidance biases. Most studies to date have used one of two reaction time tasks: The Approach/Avoidance Task (AAT; Chen \& Bargh, 1999; Solarz, 1960) or the Stimulus Response Compatibility Task (SRCT; De Houwer, Crombez, Baeyens, \& Hermans, 2001). In a typical AAT, participants are required to execute responses that result in either the approach, or avoidance, of stimulus images as fast as possible. Typically, responses on the AAT result in a zooming effect to simulate physical approach or avoidance, whereby 
approach responses are accompanied by the image increasing in size, and avoidance responses are accompanied by the image decreasing in size. Most studies have used a joystick to register responses, with participants pulling the joystick towards them to perform approach responses and pushing the joystick away from them to perform avoidance responses. However, researchers have also used computer mouse movements (Paslakis et al., 2017) or keyboard key presses (Khan \& Petróczi, 2015) to register responses. In a typical SRCT, participants are not required to execute responses that replicate approach or avoidance of stimulus images relative to themselves, but instead participants are required to move a manikin figure either towards (representing approach) or away (representing avoidance) from image stimuli via a keyboard key press.

For both the AAT and the SRCT, instructions to approach or avoid the contrasted picture categories can involve a motivationally relevant feature of the picture (e.g., whether a picture contains chocolate or vegetables) or a motivationally irrelevant feature of the picture (e.g., whether a picture is portrait or landscape in orientation). Irrelevant feature versions of the AAT are thought to more greatly index implicit biases in responding because instructions lessen conscious evaluation of picture content (Lender et al., 2018; Rinck \& Becker, 2007). Importantly, while both relevant and irrelevant feature versions of the AAT and the SRCT tasks have been used by researchers in the consumption domain, irrelevant feature versions typically yield smaller approach/avoidance bias estimates as compared to relevant feature tasks (Field et al., 2011; Kersbergen et al., 2015; Krieglmeyer \& Deutsch, 2010; Lender et al., 2018; Meule et al., 2019). For example, Lender et al. (2018) demonstrated a robust approach bias towards appetitive food images was present only under task conditions that employed relevant feature instructions, and not under conditions that employed irrelevant feature instructions. In addition, researchers have reported much 
poorer internal reliability estimates for irrelevant feature tasks when compared to relevant feature tasks measuring approach to appetitive images (Kersbergen et al., 2015). Thus, existing research indicates that AAT and SRCT tasks that adopt instructions to approach or avoid image stimuli based on motivationally relevant features provide stronger and more reliable estimates of approach/avoidance biases across individuals.

Critically, though both the AAT and SRCT tasks are commonly employed to assess approach biases and their relation to food consumption behaviour, no research has yet directly compared the reliability and the convergence of measures yielded by AAT and SRCT task designs that have been adopted by researchers in the food consumption domain. Research that has examined these questions in other individual difference domains, such as fear and problematic alcohol consumption, has observed that measures yielded from AAT and SRCT tasks are not associated in these domains. For example, Krieglmeyer and Deutsch (2010; Experiment 3) found that measures of spider avoidance bias yielded by the SRCT did not correlate with the measure yielded by the AAT across individuals who varied in fear of spiders. Similarly, Kersbergen et al. (2015) found that though alcohol approach bias scores yielded by the AAT and the SRCT each explained a small proportion of variance in problematic drinking, scores yielded by the AAT and the SRCT did not correlate. Given the absence of an association between measures yielded by the AAT and SRCT tasks in these domains is consistent with the possibility that the tasks do not measure the same process, and given the frequent use of each task by researchers seeking to understand the association between approach biases and food consumption, it is imperative that the internal reliability, convergent validity, and criterion validity of measures yielded by the AAT and SRCT in the context of food consumption are investigated. 
Thus, the aim of the present study will be to address whether (1) the AAT and SRCT paradigms provide internally reliable estimates of approach bias to unhealthy relative to healthy food stimuli, (2) the AAT and SRCT paradigms demonstrate convergence in their assessment of approach bias to unhealthy relative to healthy food stimuli, and ( 3 ) the approach bias scores to unhealthy relative to healthy food yielded by the AAT and SRCT paradigms demonstrate comparable associations with individual differences in food craving and eating behaviour. To do so, participants will complete a mouse-based AAT, a keyboardbased AAT, and an SRCT, which have each been adopted by researchers in the food consumption domain. Given that relevant feature paradigms have been demonstrated to elicit stronger and more reliable effects, as compared to irrelevant feature paradigms (Hans Phaf et al., 2014; Lender et al., 2018), the present study will employ relevant feature pardigms of each task. Each task will yield an estimate of participants' approach bias towards unhealthy food relative to healthy food. We will compare the internal reliability of each of these bias scores, the degree to which the approach bias scores converge, and the degree to which the approach bias scores from each task are concurrently associated with individual differences in food craving and eating behaviour.

\section{Method}

\section{Participants}

Participants will be recruited online via Amazon Mechanical-Turk (MTurk) in exchange for monetary compensation. Given that the stimulus set used in the study will reflect foods from an omnivorous diet, participation in the study will be restricted to individuals who follow an omnivorous diet. Participant recruitment will continue until a cohort of 120 individuals has completed the study procedure and whose data is determined 
valid for inclusion in statistical analyses (see participant exclusion criteria in Data Analysis Plan section).

A sensitivity analysis determined that $r=.26$ is the smallest effect size that could be detected with this number of participants with at least $90 \%$ power $(1-\beta=.90)$ under a onetailed test of statistical significance set at $p<.05$. This effect size is smaller than $r=.50$, commonly considered to represent a minimum acceptable level of convergent validity (Carlson \& Herdman, 2012). Thus, the sample size is considered appropriate for detecting the smallest effect size of interest for the purpose of determining whether measures yielded by behavioural tasks show a clear association with questionnaire measures of individual differences in food craving and converge enough to be considered equivalent measures of the same behavioural tendencies.

\section{Questionnaires}

\section{Demographic and Dietary Status Questionnaire}

Participants will be required to report on demographic information, including gender identity, age, dietary status (omnivorous or other), diet status (currently restricting caloric intake, or not), and current height and weight to enable computation of body mass index $\left(B M I=\mathrm{kg} / \mathrm{m}^{2}\right)$.

\section{Food Cravings Questionnaire-Trait-reduced}

Dispositional tendency to experience general food cravings will be assessed using the English language version of the 15-item Food Cravings Questionnaire-Trait-reduced (FCQ-Tr; Meule et al., 2014). Items are rated on a five-point Likert scale ( 1 = strongly disagree, $5=$ strongly agree) with higher scores indicating higher trait food craving. The English language version of the FCQ-T-r has demonstrated excellent internal consistency reliability and good 
convergent validity (Hormes \& Meule, 2016). The internal consistency of this measure in the current study will be reported.

\section{Dutch Eating Behaviour Questionnaire}

Trait eating behaviours will be assessed using the Dutch Eating Behavior Questionnaire (DEBQ; van Strien et al., 1986). These eating behaviours include: Restrained Eating (10 items), Emotional Eating (13 items), and External Eating (10 items). Respondents rate each item on a five-point Likert scale, ranging from 1 (never) to 5 (very often). For each subscale, an average score is computed, with higher scores indicating greater levels of engagement in restrained, emotional, or external eating behaviours. The three subscales have demonstrated excellent internal consistency, factorial validity, and predictive validity with eating behaviours (van Strien et al., 1986, 2007, 2012). The internal consistency of this measure in the current study will be reported.

\section{Stimulus Images}

Eighty images displaying food items were selected from the Extended Food-pics Database (Blechert et al., 2019) for use in the approach bias assessment tasks. Half of the images (40) depict healthy foods, while the other half (40) depict unhealthy foods. In order to objectively differentiate healthy and unhealthy foods, two key nutritional components (i.e., sugar and fat content grams/100g) were estimated for each food image using the FoodData Central Database of the United States Department of Agriculture (https://fdc.nal.usda.gov/). In line with Australian Dietary Guidelines (National Health and Medical Research Council, 2013), a food item was classified as healthy if its sugar and fat content did not exceed $10 \mathrm{~g}$ per $100 \mathrm{~g}$, unless most of the sugar was coming from fruit, and unhealthy if it did not meet this criterion. As expected, this criterion resulted in unhealthy food images depicting foods that are, on average, significantly higher in calorie content than 
foods depicted in healthy food images $, t(40.48)=3.73, p<.001$. Healthy and unhealthy foods did not differ in brightness, complexity, and recognisability (all $p s>.06$ ). An additional set of 12 images was selected for use in practice trials of the approach bias assessment tasks.

\section{Approach Bias Assessment Tasks}

Participants will complete three approach bias assessment tasks that reflect task designs used in the investigation of the relationship between approach biases and craving and eating behaviour. These tasks are the Stimulus-Response Compatibility Task, the Approach-Avoidance Task - Arm Movement Response, and the Approach-Avoidance Task Key Press Response. Features that are common across these tasks will be described first, followed by a description of those features unique to each task.

\section{Features common across approach bias assessment tasks}

Each approach bias assessment task will consist of two blocks presented in a fixed order. The first block, labelled the approach unhealthy foods block, presents trials in which participants are required to execute task specific responses that result in approach towards unhealthy foods and avoidance of healthy foods. The second block, labelled the approach healthy foods block, presents trials in which participants are required to execute task specific responses that result in approach towards healthy foods and avoidance of unhealthy foods. Each assessment block contains 80 trials and is immediately preceded by a block of instructions and 12 practice trials that provide feedback on the correctness of each response. Images used in each block are derived from the Assessment Stimulus Set described earlier, with each image randomly presented once per block. All trials are presented on a black background. 
For each participant at the conclusion of each task, an index of behavioural approach will be computed by subtracting the median response latency of correct approach trials from the median response latency of correct avoidance trials, separately for trials depicting unhealthy foods and for trials depicting healthy foods. Thus, each index will reflect the relative speeding for participants to execute responses that approach each food type, relative to responses that avoid each food type. Next, an Unhealthy Food Approach Bias Index score will be computed by subtracting the latter value (healthy foods) from the former value (unhealthy foods). Thus, larger index scores on the Unhealthy Food Approach Bias Index represent a greater bias to approach, relative to avoid, unhealthy foods as compared to healthy foods. This computation can be formally expressed as:

\section{Unhealthy Food Approach Bias Index =}

[Median latency for avoiding unhealthy foods - Median latency for approaching unhealthy foods] - [Median latency for avoiding healthy foods - Median latency for approaching healthy foods]

\section{Stimulus-Response Compatibility Task}

Each trial of the Stimulus-Response Compatibility Task (SRC) will start with a 1000ms black screen. Next, a single picture $(80 \mathrm{~mm} \times 80 \mathrm{~mm})$ will be presented in the centre of the screen, and a white stick figure manikin $(55 \mathrm{~mm} \times 55 \mathrm{~mm})$ will be presented either above or below the picture. In approach unhealthy foods blocks, participants will be required to move the manikin towards images containing unhealthy foods and away from images containing healthy foods, using either the Y-key (move manikin up) or the B-key (move manikin down) of a standard QWERTY-keyboard. In approach healthy foods blocks, participants will be required to move the manikin towards images containing healthy foods and away from images containing unhealthy foods, using the same response keys. Across all blocks, the four 
different trial types following from combinations of the manikin's starting position (above versus below the image) and the image content (healthy versus unhealthy food) will be presented in random order but with equal frequency. Upon a correct response the manikin will move in the appropriate direction. An incorrect response will be immediately followed by a five second delay with the text "incorrect response" displayed on screen. The time taken for participants to execute the key press response following presentation of the image, and accuracy of the response, will be recorded by the task.

\section{Approach-Avoidance Task - Arm Movement Response}

Each trial of the Approach-Avoidance Task with arm movement response will start with a 1000 ms black screen. Next, a red ' $X$ ' is presented in the middle of the screen. Participants are required to click on the $\mathrm{X}$ with their mouse pointer to initiate each trial. Immediately following, the ' $X$ ' is replaced by a square picture sized to be $50 \%$ of the screen height. In approach unhealthy foods blocks, participants will be instructed to execute an approach movement by contracting their arm to pull the mouse towards themselves in response to images containing unhealthy foods, and to execute an avoidance movement by extending their arm to push the mouse away from themselves in response to images containing healthy foods. In approach healthy foods blocks, participants will be instructed to execute the approach movement in response to images containing healthy foods and execute the avoidance movement in response to images containing unhealthy foods. To simulate change in the physical distance between the picture and participant, the size of the image will increase or decrease in response to the movement of the mouse. Specifically, when participants pull the mouse towards themselves the picture will progressively increase in size and when participants push the mouse away from themselves the picture will progressively reduce in size. The trial concludes when the image has either increased to the 
total height of the screen or has reduced to $10 \%$ of the total height of the screen. Trials on which participants make incorrect responses will be immediately followed by a five second delay with the text "incorrect final mouse movement" displayed on screen. At the conclusion of each trial, the time taken for participants to complete the mouse movement response following presentation of the image, and accuracy of the response, will be recorded by the task.

\section{Approach-Avoidance Task - Key Press Response}

Each trial of the Approach-Avoidance Task with key press response will be identical to the design of the arm movement response task, except that participants will be required to execute responses using a keyboard key press rather than movement of the mouse.

Specifically, in approach unhealthy foods blocks participants will be required to execute an approach response by pressing the down-arrow key on the keyboard when images containing unhealthy foods appear on the screen, and to execute an avoidance response by pressing the up-arrow key on the keyboard when images containing healthy foods appear on the screen. In approach healthy foods blocks, participants will be required to execute the approach response when images containing healthy foods appear on the screen and execute the avoidance response when images containing unhealthy foods appear on the screen. To simulate change in the physical distance between the picture and participant, the size of the image will increase or decrease in response to the key press. Specifically, when participants press the down-arrow key the picture will progressively increase in size, and when participants press the up-arrow key the picture will progressively reduce in size. An incorrect response will be immediately followed by a five second delay with the text "incorrect response" displayed on screen. At the conclusion of each trial, the time taken for 
participants to execute the key press response following presentation of the image, and accuracy of the response, will be recorded by the task.

\section{Procedure}

All procedures necessary to conduct this research have been approved by the Human Research Ethics Committee at the University of Western Australia. The procedure will be delivered via the internet to participants' personal computers using the Inquisit Web experiment platform. Prior to recruitment, individuals will be informed that the study seeks to examine patterns of cognitive processing across different assessment conditions and will be informed of the requirements involved in participation. Upon accepting the invitation to participate in the study participants will be directed to a URL that hosts the experiment assessments. Participants will first be required to provide informed consent and demographic information before then completing the questionnaires. Participants will next complete the approach bias assessment tasks. Tasks will be delivered in a counter balanced order such that an equal number of participants completes the tasks in each possible combination. The tasks will be programmed to maintain equivalent spatial parameters amongst visual stimuli across different screen sizes and resolutions. The length of the procedure is anticipated to be less than 60 minutes. Upon completion of the tasks, participants will be debriefed.

\section{Data Analysis Plan}

\section{Participant and Data Exclusion Criteria}

Participants will be excluded from data analysis if they do not complete the entire study procedure or complete any part of the procedure more than once. As the study aims to compare indices of task performance between behavioural assessment tasks it was determined important that computed indices are conducted upon data that is drawn from 
participants who demonstrate adherence to task instructions across all tasks. Thus, a listwise exclusion method will exclude remaining participants if they demonstrate incorrect responding on more than $50 \%$ of trials on any task, or subsequently an incorrect response rate that is more than 2.58 standard deviations below the mean rate for that task across all participants.

\section{Statistical Analyses}

The objectives of the statistical analyses will first be to determine to determine the internal reliability of response measures recorded by each task. Next, statistical analyses will determine the convergence of approach bias measures yielded across the assessment tasks, followed by determination of the association between observed bias scores and measures of individual differences in food craving and eating behaviours. Statistical significance will be determined using an alpha criterion of $p<.05$.

\section{Descriptive statistics}

Descriptive statistics will be reported for self-report measures, and measures of total response accuracy, median response latency for critical trial conditions, and Unhealthy Food Approach Bias Index scores recorded under each approach bias assessment task. Descriptive statistics will report means, standard deviations, and proportions as relevant.

\section{Assessment of internal reliability of bias indices yielded by behavioural tasks}

A measure of internal consistency will be computed for each assessment task using a random permutation split-half correlation approach. This approach splits each participant's trial data into two randomly selected halves before computing the Unhealthy Food Approach Bias Index for each half. The approach then computes the Pearson correlation coefficient between these indices across all participants, including the Spearman-Brown formula adjustment. This process is repeated across 5000 randomly selected split-half trial 
combinations. The measure of internal consistency is determined by computing the mean and $95 \%$ confidence interval of the distribution of correlations coefficients observed in the samples.

\section{Assessment of convergence of bias index scores}

Convergence between the Unhealthy Food Approach Bias Index scores yielded by each assessment task will be examined to determine whether the assessment tasks plausibly reflect the same approach action tendencies. Convergence will be examined via the statistical association between indices yielded by each assessment task. For analyses in which both variables are normally distributed associations will be examined by using Pearson correlation coefficients, and for analyses in which one or more variables are not normally distributed associations will be examined by using Spearman correlations. Given the presence of clear directional hypotheses predicting positive associations between measures of Unhealthy Food Approach Bias Index scores yielded by each task, statistical significance for these analyses will be determined using one-tailed tests.

\section{Associations with individual difference measures}

We will then seek to determine whether the Unhealthy Food Approach Bias Index scores yielded by each assessment task are associated with individual differences in selfreported food craving (FCQ-T-r) and eating behaviours (DEBQ subscales). For analyses in which both variables are normally distributed associations will be determined by using Pearson correlation coefficients, and for analyses in which one or more variables are not normally distributed associations will be determined by using Spearman correlations. Given the presence of clear directional hypotheses predicting positive associations between level of food craving and eating behaviours and Unhealthy Food Approach Bias Index scores, statistical significance for these analyses will be determined using one-tailed tests. 


\section{Timeline for completion}

This study will commence as soon as Stage 1 approval is granted. It is anticipated that participant testing for the study will be completed within 8 weeks from the date of receiving Stage 1 approval. 


\section{References}

Blechert, J., Lender, A., Polk, S., Busch, N. A., \& Ohla, K. (2019). Food-pics_extended-an image database for experimental research on eating and appetite: Additional images, normative ratings and an updated review. Frontiers in Psychology. https://doi.org/10.3389/fpsyg.2019.00307

Brockmeyer, T., Hahn, C., Reetz, C., Schmidt, U., \& Friederich, H. C. (2015). Approach bias and cue reactivity towards food in people with high versus low levels of food craving. Appetite, 95, 197-202. https://doi.org/10.1016/j.appet.2015.07.013

Carlson, K. D., \& Herdman, A. O. (2012). Understanding the impact of convergent validity on research results. Organizational Research Methods, 15(1), 17-32. https://doi.org/10.1177/1094428110392383

Chen, M., \& Bargh, J. A. (1999). Consequences of automatic evaluation: Immediate behavioral predispositions to approach or avoid the stimulus. In Personality and Social Psychology Bulletin. https://doi.org/10.1177/0146167299025002007

De Houwer, J., Crombez, G., Baeyens, F., \& Hermans, D. (2001). On the generality of the affective Simon effect. Cognition and Emotion. https://doi.org/10.1080/02699930125883

Elliot, A. J. (2006). The Hierarchical Model of Approach-Avoidance Motivation. Motivation and Emotion, 30(2), 111-116. https://doi.org/10.1007/s11031-006-9028-7

Field, M., Caren, R., Fernie, G., \& De Houwer, J. (2011). Alcohol approach tendencies in heavy drinkers: Comparison of effects in a relevant stimulus-response compatibility task and an approach/avoidance Simon task. Psychology of Addictive Behaviors, 25(4), 697-701. https://doi.org/10.1037/a0023285

Fricke, K., \& Vogel, S. (2020). How interindividual differences shape approach-avoidance behavior: Relating self-report and diagnostic measures of interindividual differences to behavioral measurements of approach and avoidance. In Neuroscience and Biobehavioral Reviews (Vol. 111, pp. 30-56). Elsevier Ltd. https://doi.org/10.1016/j.neubiorev.2020.01.008

Hans Phaf, R., Mohr, S. E., Rotteveel, M., \& Wicherts, J. M. (2014). Approach, avoidance, and affect: A meta-analysis of approach-avoidance tendencies in manual reaction time tasks. Frontiers in Psychology, 5(MAY). https://doi.org/10.3389/fpsyg.2014.00378

Hormes, J. M., \& Meule, A. (2016). Psychometric properties of the English Food Cravings Questionnaire-Trait-reduced (FCQ-T-r). Eating Behaviors. https://doi.org/10.1016/j.eatbeh.2015.11.011

Kakoschke, N., Albertella, L., Lee, R. S. C., \& Wiers, R. W. (2019). Assessment of Automatically Activated Approach-Avoidance Biases Across Appetitive Substances. Current Addiction Reports, 6(3), 200-209. https://doi.org/10.1007/s40429-019-00254-2

Kakoschke, N., Kemps, E., \& Tiggemann, M. (2017a). Approach bias modification training and consumption: A review of the literature. In Addictive Behaviors. https://doi.org/10.1016/j.addbeh.2016.08.007

Kakoschke, N., Kemps, E., \& Tiggemann, M. (2017b). Differential effects of approach bias and eating style on unhealthy food consumption in overweight and normal weight women. Psychology and Health. https://doi.org/10.1080/08870446.2017.1327587

Kersbergen, I., Woud, M. L., \& Field, M. (2015). The validity of different measures of automatic alcohol action tendencies. Psychology of Addictive Behaviors.

https://doi.org/10.1037/adb0000009 
Khan, S., \& Petróczi, A. (2015). Stimulus-response compatibility tests of implicit preference for food and body image to identify people at risk for disordered eating: A validation study. Eating Behaviors, 16, 54-63. https://doi.org/10.1016/j.eatbeh.2014.10.015

KriegImeyer, R., \& Deutsch, R. (2010). Comparing measures of approach-avoidance behaviour: The manikin task vs. two versions of the joystick task. Cognition and Emotion, 24(5), 810-828. https://doi.org/10.1080/02699930903047298

Lang, P. J. (1995). The Emotion Probe: Studies of Motivation and Attention. American Psychologist. https://doi.org/10.1037/0003-066X.50.5.372

Lender, A., Meule, A., Rinck, M., Brockmeyer, T., \& Blechert, J. (2018). Measurement of food-related approach-avoidance biases: Larger biases when food stimuli are task relevant. Appetite, 125(February), 42-47. https://doi.org/10.1016/j.appet.2018.01.032

Loijen, A., Vrijsen, J. N., Egger, J. I. M., Becker, E. S., \& Rinck, M. (2020). Biased approachavoidance tendencies in psychopathology: A systematic review of their assessment and modification. Clinical Psychology Review, 77, 101825. https://doi.org/10.1016/j.cpr.2020.101825

Mehl, N., Mueller-Wieland, L., Mathar, D., \& Horstmann, A. (2018). Retraining automatic action tendencies in obesity. Physiology and Behavior. https://doi.org/10.1016/j.physbeh.2018.03.031

Meule, A., Hermann, T., \& Kübler, A. (2014). A short version of the food cravings questionnaire-trait: The FCQ-T-reduced. Frontiers in Psychology. https://doi.org/10.3389/fpsyg.2014.00190

Meule, A., Lender, A., Richard, A., Dinic, R., \& Blechert, J. (2019). Approach-avoidance tendencies towards food: Measurement on a touchscreen and the role of attention and food craving. Appetite, 137(March), 145-151. https://doi.org/10.1016/j.appet.2019.03.002

National Health and Medical Research Council. (2013). Australian Dietary Guidelines. National Health and Medical Research Council. https://www.nhmrc.gov.au/adg

Paslakis, G., Kühn, S., Grunert, S., \& Erim, Y. (2017). Explicit and implicit approach vs. Avoidance tendencies towards high vs. Low calorie food cues in patients with obesity and active binge eating disorder. Nutrients. https://doi.org/10.3390/nu9101068

Rinck, M., \& Becker, E. S. (2007). Approach and avoidance in fear of spiders. Journal of Behavior Therapy and Experimental Psychiatry, 38(2), 105-120. https://doi.org/10.1016/j.jbtep.2006.10.001

Schmidt, R., Sebert, C., Kösling, C., Grunwald, M., Hilbert, A., Hübner, C., \& Schäfer, L. (2018). Neuropsychological and Neurophysiological Indicators of General and FoodSpecific Impulsivity in Children with Overweight and Obesity: A Pilot Study. Nutrients, 10(12), 1983. https://doi.org/10.3390/nu10121983

Solarz, A. K. (1960). Latency of instrumental responses as a function of compatibility with the meaning of eliciting verbal signs. Journal of Experimental Psychology. https://doi.org/10.1037/h0047274

van Strien, T., Frijters, J. E. R., Bergers, G. P. A., \& Defares, P. B. (1986). The Dutch Eating Behavior Questionnaire (DEBQ) for assessment of restrained, emotional, and external eating behavior. International Journal of Eating Disorders, 5(2), 295-315. https://doi.org/10.1002/1098-108X(198602)5:2<295::AID-EAT2260050209>3.0.CO;2-T van Strien, T., Peter Herman, C., \& Anschutz, D. (2012). The predictive validity of the DEBQexternal eating scale for eating in response to food commercials while watching television. International Journal of Eating Disorders, 45(2), 257-262. 
https://doi.org/10.1002/eat.20940

van Strien, T., Peter Herman, C., Engels, R. C. M. E., Larsen, J. K., \& van Leeuwe, J. F. J.

(2007). Construct validation of the Restraint Scale in normal-weight and overweight females. Appetite, 49(1), 109-121. https://doi.org/10.1016/j.appet.2007.01.003

Blechert, J., Lender, A., Polk, S., Busch, N. A., \& Ohla, K. (2019). Food-pics_extended-an image database for experimental research on eating and appetite: Additional images, normative ratings and an updated review. Frontiers in Psychology. https://doi.org/10.3389/fpsyg.2019.00307

Brockmeyer, T., Hahn, C., Reetz, C., Schmidt, U., \& Friederich, H. C. (2015). Approach bias and cue reactivity towards food in people with high versus low levels of food craving. Appetite, 95, 197-202. https://doi.org/10.1016/j.appet.2015.07.013

Carlson, K. D., \& Herdman, A. O. (2012). Understanding the impact of convergent validity on research results. Organizational Research Methods, 15(1), 17-32. https://doi.org/10.1177/1094428110392383

Chen, M., \& Bargh, J. A. (1999). Consequences of automatic evaluation: Immediate behavioral predispositions to approach or avoid the stimulus. In Personality and Social Psychology Bulletin. https://doi.org/10.1177/0146167299025002007

De Houwer, J., Crombez, G., Baeyens, F., \& Hermans, D. (2001). On the generality of the affective Simon effect. Cognition and Emotion. https://doi.org/10.1080/02699930125883

Elliot, A. J. (2006). The Hierarchical Model of Approach-Avoidance Motivation. Motivation and Emotion, 30(2), 111-116. https://doi.org/10.1007/s11031-006-9028-7

Field, M., Caren, R., Fernie, G., \& De Houwer, J. (2011). Alcohol approach tendencies in heavy drinkers: Comparison of effects in a relevant stimulus-response compatibility task and an approach/avoidance Simon task. Psychology of Addictive Behaviors, 25(4), 697-701. https://doi.org/10.1037/a0023285

Fricke, K., \& Vogel, S. (2020). How interindividual differences shape approach-avoidance behavior: Relating self-report and diagnostic measures of interindividual differences to behavioral measurements of approach and avoidance. In Neuroscience and Biobehavioral Reviews (Vol. 111, pp. 30-56). Elsevier Ltd. https://doi.org/10.1016/j.neubiorev.2020.01.008

Hans Phaf, R., Mohr, S. E., Rotteveel, M., \& Wicherts, J. M. (2014). Approach, avoidance, and affect: A meta-analysis of approach-avoidance tendencies in manual reaction time tasks. Frontiers in Psychology, 5(MAY). https://doi.org/10.3389/fpsyg.2014.00378

Hormes, J. M., \& Meule, A. (2016). Psychometric properties of the English Food Cravings Questionnaire-Trait-reduced (FCQ-T-r). Eating Behaviors. https://doi.org/10.1016/j.eatbeh.2015.11.011

Kakoschke, N., Albertella, L., Lee, R. S. C., \& Wiers, R. W. (2019). Assessment of Automatically Activated Approach-Avoidance Biases Across Appetitive Substances. Current Addiction Reports, 6(3), 200-209. https://doi.org/10.1007/s40429-019-00254-2

Kakoschke, N., Kemps, E., \& Tiggemann, M. (2017a). Approach bias modification training and consumption: A review of the literature. In Addictive Behaviors.

https://doi.org/10.1016/j.addbeh.2016.08.007

Kakoschke, N., Kemps, E., \& Tiggemann, M. (2017b). Differential effects of approach bias and eating style on unhealthy food consumption in overweight and normal weight women. Psychology and Health. https://doi.org/10.1080/08870446.2017.1327587

Kersbergen, I., Woud, M. L., \& Field, M. (2015). The validity of different measures of 
automatic alcohol action tendencies. Psychology of Addictive Behaviors. https://doi.org/10.1037/adb0000009

Khan, S., \& Petróczi, A. (2015). Stimulus-response compatibility tests of implicit preference for food and body image to identify people at risk for disordered eating: A validation study. Eating Behaviors, 16, 54-63. https://doi.org/10.1016/j.eatbeh.2014.10.015

KriegImeyer, R., \& Deutsch, R. (2010). Comparing measures of approach-avoidance behaviour: The manikin task vs. two versions of the joystick task. Cognition and Emotion, 24(5), 810-828. https://doi.org/10.1080/02699930903047298

Lang, P. J. (1995). The Emotion Probe: Studies of Motivation and Attention. American Psychologist. https://doi.org/10.1037/0003-066X.50.5.372

Lender, A., Meule, A., Rinck, M., Brockmeyer, T., \& Blechert, J. (2018). Measurement of food-related approach-avoidance biases: Larger biases when food stimuli are task relevant. Appetite, 125(February), 42-47. https://doi.org/10.1016/j.appet.2018.01.032

Loijen, A., Vrijsen, J. N., Egger, J. I. M., Becker, E. S., \& Rinck, M. (2020). Biased approachavoidance tendencies in psychopathology: A systematic review of their assessment and modification. Clinical Psychology Review, 77, 101825. https://doi.org/10.1016/j.cpr.2020.101825

Mehl, N., Mueller-Wieland, L., Mathar, D., \& Horstmann, A. (2018). Retraining automatic action tendencies in obesity. Physiology and Behavior. https://doi.org/10.1016/j.physbeh.2018.03.031

Meule, A., Hermann, T., \& Kübler, A. (2014). A short version of the food cravings questionnaire-trait: The FCQ-T-reduced. Frontiers in Psychology. https://doi.org/10.3389/fpsyg.2014.00190

Meule, A., Lender, A., Richard, A., Dinic, R., \& Blechert, J. (2019). Approach-avoidance tendencies towards food: Measurement on a touchscreen and the role of attention and food craving. Appetite, 137(March), 145-151.

https://doi.org/10.1016/j.appet.2019.03.002

National Health and Medical Research Council. (2013). Australian Dietary Guidelines. National Health and Medical Research Council. https://www.nhmrc.gov.au/adg

Paslakis, G., Kühn, S., Grunert, S., \& Erim, Y. (2017). Explicit and implicit approach vs. Avoidance tendencies towards high vs. Low calorie food cues in patients with obesity and active binge eating disorder. Nutrients. https://doi.org/10.3390/nu9101068

Rinck, M., \& Becker, E. S. (2007). Approach and avoidance in fear of spiders. Journal of Behavior Therapy and Experimental Psychiatry, 38(2), 105-120. https://doi.org/10.1016/j.jbtep.2006.10.001

Schmidt, R., Sebert, C., Kösling, C., Grunwald, M., Hilbert, A., Hübner, C., \& Schäfer, L. (2018). Neuropsychological and Neurophysiological Indicators of General and FoodSpecific Impulsivity in Children with Overweight and Obesity: A Pilot Study. Nutrients, 10(12), 1983. https://doi.org/10.3390/nu10121983

Solarz, A. K. (1960). Latency of instrumental responses as a function of compatibility with the meaning of eliciting verbal signs. Journal of Experimental Psychology. https://doi.org/10.1037/h0047274

van Strien, T., Frijters, J. E. R., Bergers, G. P. A., \& Defares, P. B. (1986). The Dutch Eating Behavior Questionnaire (DEBQ) for assessment of restrained, emotional, and external eating behavior. International Journal of Eating Disorders, 5(2), 295-315. https://doi.org/10.1002/1098-108X(198602)5:2<295::AID-EAT2260050209>3.0.CO;2-T van Strien, T., Peter Herman, C., \& Anschutz, D. (2012). The predictive validity of the DEBQ- 
external eating scale for eating in response to food commercials while watching television. International Journal of Eating Disorders, 45(2), 257-262. https://doi.org/10.1002/eat.20940

van Strien, T., Peter Herman, C., Engels, R. C. M. E., Larsen, J. K., \& van Leeuwe, J. F. J. (2007). Construct validation of the Restraint Scale in normal-weight and overweight females. Appetite, 49(1), 109-121. https://doi.org/10.1016/j.appet.2007.01.003 